\title{
On Efficiency Group Multicasting Algorithm with Multiple Minimum Steiner Trees
}

\author{
Moonseong $\mathrm{Kim}^{1}$, Minseok Kang ${ }^{2}$, Hyunseung $\mathrm{Choo}^{1}$, \\ Jong S. Yang ${ }^{3}$, and Young-Cheol Bang ${ }^{2, \star}$ \\ ${ }^{1}$ School of Info. and Comm. Engineering, Sungkyunkwan University, Korea \\ \{moonseong, choo\}@ece.skku.ac.kr \\ ${ }^{2}$ Dep. of Computer Engineering, Korea Polytechnic University, Korea \\ $\{\mathrm{k} 19 \mathrm{~m} 81 \mathrm{~s}$, ybang\}@kpu.ac.kr \\ ${ }^{3}$ Korea Institute of Industrial Technology Evaluation and Planning, Korea \\ yjs@mail.itep.re.kr
}

\begin{abstract}
In this paper, we study the problem of constructing minimum cost group multicast trees with bandwidth reservations. Our algorithm uses multiple candidate paths to select a path from source to each destination member in each multicast tree construction. We show that our proposed algorithm performs better in terms of total tree cost for real life networks over well-known algorithm GKMB. The enhancement is up to about $10 \% \sim 25 \%$ in terms of normalized surcharge for the GKMB tree cost.
\end{abstract}

Keywords: Group Multicast Routing Algorithm, Minimum Steiner Trees, Group KMB (GKMB) Algorithm, and Minimum Cost Multicast Tree (MCMT) Algorithm.

\section{Introduction}

With the emergence of real-time applications, such as video conferences and online games, and peer-to-peer (P2P) applications, and other content distribution networks, a group of entities may exchange data among themselves. A network layer may provide support for these applications by creating a set of multicast routing trees simultaneously. The problem of finding a set of multicast trees where every member in a group is a terminal as well as a source node is called the Group Multicast Routing Problem (GMRP). The routing algorithm must construct a multicast tree for each member node with each tree rooted at the member and spanning all other member nodes simultaneously.

Jia and Wang proposed a group multicast routing algorithm, Group KMB (GKMB) [1, that is based on KMB algorithm. The KMB heuristic was proposed by Kou, Markowsky, and Berman 2. KMB applies Prim's minimum spanning tree algorithm to the complete distance graph, where the complete distance graph is a graph that contains Steiner points (multicast members) and has an

\footnotetext{
^ Corresponding author.
} 
edge between every pair of nodes representing the shortest path between them. $\mathrm{KMB}$ is very well-known heuristic algorithm in minimum Steiner tree problem.

Recently, Bang et al. proposed the Minimum Cost Multicast Tree (MCMT) algorithm [3] to create minimum Steiner tree. MCMT is proved that it has the nearest optimal tree cost than other algorithms, are known to be the best heuristic. Although KMB algorithm has the nearest optimal solution, we strongly believe that MCMT outperforms KMB in terms of tree cost. Therefore, in this paper, we propose an algorithm, called Group MCMT (GMCMT), that is based on the adaptation of MCMT algorithm. Also, simulation studies show that our algorithm outperforms GKMB.

The subsequent sections of this paper are organized as follows. In Section 2, the network model and previous algorithms are described. Section 3 presents details of our algorithm. Section 4 shows the results of simulation studies. This paper is finally concluded in Section 5 .

\section{Preliminaries}

\subsection{Network Model}

The source node of a multicasting is assumed to know all the information needed to construct the multicast tree. A network is modeled as a directed weighted graph $G=(V, E)$ with node set $V$ and edge (link or arc) set $E$. An edge $e \in E$ from $u \in V$ to $v \in V$ is represented by $e=(u, v)$. We define a path as sequence of links such that $\left(v_{0}, v_{1}\right),\left(v_{1}, v_{2}\right), \ldots,\left(v_{i-1}, v_{i}\right)$, belongs to $E$. Let $P\left(v_{0}, v_{i}\right)=\left\{\left(v_{0}, v_{1}\right),\left(v_{1}, v_{2}\right), \ldots,\left(v_{i-1}, v_{i}\right)\right\}$ denote the path from node $v_{0}$ to node $v_{i}$. The path contains the nodes $v_{0}, v_{1}, \ldots, v_{i}$ and the edges $\left(v_{0}, v_{1}\right),\left(v_{1}, v_{2}\right), \ldots,\left(v_{i-1}, v_{i}\right)$. Each link $e=(u, v) \in E$ is associated with two parameters, namely link cost $c(e) \geq 0$ and available link bandwidth $b(e) \geq 0$. They are asymmetric in general. The cost of a link can be associated with the utilization of the link. A higher utilization is represented by a higher link cost. The path cost of path $P(s, m)$ is $\phi_{C}(P)=\sum_{e \in P} c(e)$ and the tree cost of tree $T$ is given by $\phi_{C}(T)=\sum_{e \in T} c(e)$.

For a given a network graph $G(V, E)$, let a multicasting group be $D \subset V$. $D=\left\{m_{1}, m_{2}, \ldots, m_{k}\right\}$ is a group of nodes in $G$, where $|D|=k$. For each node $m_{i} \in D$, multicast member node $m_{i}$ has a bandwidth requirements of $B_{i}$ units. The bandwidth requirement $B_{i}$ of each node in $D$ is specified by the user. The GMRP is that of finding a set of directed routing trees $\left\{T_{1}, T_{2}, \ldots, T_{k}\right\}$, one for each member of group $D$ which satisfy the follows:

$$
\text { minimize }\left\{\sum_{i=1}^{k} \sum_{e \in T_{i}} c(e) \chi_{e}^{i},{ }^{\forall} e \in E\right\}
$$

subject to

$$
\sum_{i=1}^{k} B_{i} \chi_{e}^{i} \leq b(e), \text { where } \chi_{e}^{i}= \begin{cases}1 & \text { if } e \in E_{i} \\ 0 & \text { otherwise }\end{cases}
$$


for each tree $T_{i}=\left(V, E_{i}\right)$, where $E_{i} \subset E$, represents the tree rooted at node $m_{i} \in D$ that spans all members in $D$.

The objective function ensures that the total cost of generated trees is minimized under constraint (1) is to ensure that the total bandwidth utilized on each link does not exceed its available bandwidth. A set of trees $\left\{T_{1}, T_{2}, \ldots, T_{k}\right\}$ which satisfy constraint (11) is called a feasible solution to the GMRP. A link is called to be saturated if the difference between its available bandwidth and its allocated bandwidth is less than the amount of bandwidth required by a user.

Variations of the GMRP have been investigated in the literature, including GMRP under bandwidth constraints, GMRP under delay constraints, GMRP protocols, and the static and dynamic GMRP. GMRP under QoS constraints has drawn attention with the increasing development of real-time multimedia applications, such as video-conference and on-line games. In this paper, we focus on the bandwidth constrained GMRP.

\subsection{Group KMB Algorithm}

Jia and Wang proposed Group KMB (GKMB) [1] using KMB [2] as the single source multicast routing algorithm. This algorithm constructs the set of multicast trees by sequentially invoking a single source multicast tree algorithm over the multicast member nodes. If any links in the network become saturated, i.e., overloaded, in the process, it implies that some multicast trees constructed previously which use these links have to release them and take alternative paths.

The algorithm KMB by Kou, Markowsky, and Berman is a minimum spanning tree based algorithm. Doar and Leslie report that KMB usually achiving 5\% of the optimal for a large number of realistic instances [4]. KMB algorithm is illustrated in Fig. 1. To find a tree, KMB starts with constructing the complete distance network $G^{\prime}=\left(V^{\prime}, E^{\prime}\right)$ induced by $D$ where, $V^{\prime}$ contains source node and destination nodes $D$ only, and $E^{\prime}$ is a set of links connecting nodes in $V^{\prime}$ for each other. In next step, a minimum spanning tree $\mathrm{T}$ of $G^{\prime}$ is determined. After then, a subgraph $G_{s}$ is constructed by replacing each link $(i, j)$ of $\mathrm{T}$ with its actual corresponding minimum cost path from $i$ to $j$ in $G$. If there exist several minimum cost paths, pick an arbitrary one. Next step is to find the minimum spanning tree $T^{\prime}$ of $G_{s}$. In final step, delete from $T^{\prime}$ all unnecessary nodes and corresponding links. Then the resulting tree is a KMB tree. Fig. 1(b) shows the complete graph from the given network Fig. 1(a) and the minimal spanning tree. Fig. 1(c) represents KMB tree by replacing each edge in the spanning tree by its corresponding shortest path in the given network.

In GKMB algorithm, a multicast tree rooted at $v$ is constructed for each $v \in D$ using KMB algorithm. When two or more trees compete for a saturated link, it would simply imply that some of these trees would have to use alternative links to get to the other member nodes in the trees. The difference in cost between the original tree and the alternative tree is known as the alternative overhead. The tree with the least alternative overhead will be forced to give up this link and take the alternative link. GKMB has shown that the algorithm runs in time $O\left(k^{3} n^{2}\right)$ with $|D|=k$ and $|V|=n$. 


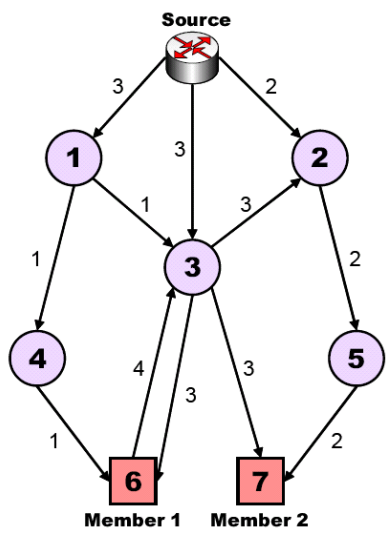

(a) A given network
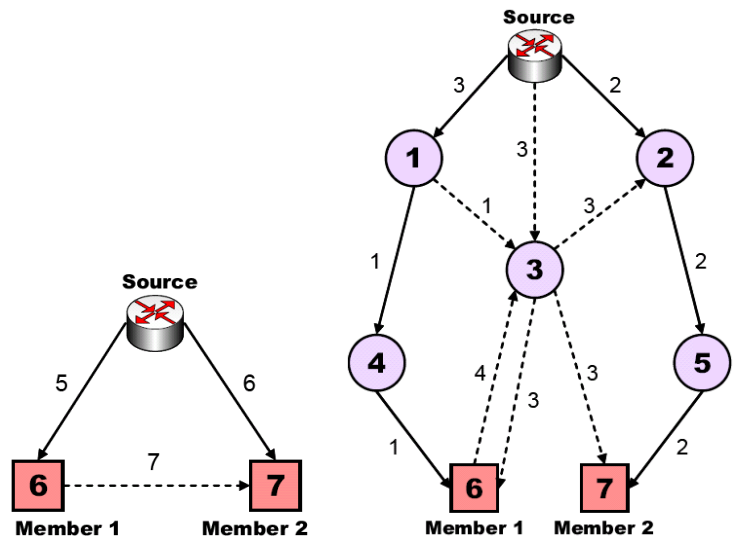

(b) The complete (c) A multicast tree based on graph and the minimal KMB spanning tree

Fig. 1. e.g., KMB algorithm

\section{The Proposed Algorithm}

\subsection{MCMT Algorithm}

Recently, Bang et al. proposed the Minimum Cost Multicast Tree (MCMT) algorithm 3 to create Steiner tree. MCMT is based on the Modified Dijkstra's Shortest Path (MDSP) algorithm to select all the minimum cost paths from source to each destination in $G$ [5] and Prim's minimum spanning tree [6].

In Fig. 2, the sub-graph $G_{m_{j}}^{\alpha}$ such that $G_{m_{j}}^{\alpha}$ is constructed by merging all the shortest paths from $\alpha \in V$ to each $m_{j} \in D$, where $G_{m_{j}}^{\alpha}$ can be constructed using MDSP. Thus, any path of $G_{m_{j}}^{\alpha}$ is a minimum cost path, also $G_{m_{j}}^{\alpha}$ is an acyclic graph. Let $T$ be a set of nodes that constitute a tree we want to define, and be empty initially. Let $G^{\prime}=\left(V^{\prime}, E^{\prime}\right)$ be sub-graph of $G$ with $V^{\prime} \subseteq V$ and $E^{\prime} \subseteq E$, where $G^{\prime}=G^{\prime} \cup G_{m_{j}}^{\alpha}$ with $G^{\prime}=\emptyset$ initially. Then, the conceptual main idea of MCMT is as follows; select $\left(s, m_{k}\right)$ pair, called $\left(s, m_{k}\right)_{\text {min }}$ such that $\phi_{C}\left(P\left(s, m_{k}\right)\right)$ is minimum among all $\left(s, m_{i}\right)$ pairs with $m_{i} \in D$ where, $s$ is a source of the multicast communication at initial step. If $G^{\prime} \neq \emptyset$, find $\left(\alpha, m_{j}\right)_{\min }$ pair with $\alpha \in V^{\prime}$ and $m_{j} \in D$. If $\alpha$ is not in $T$ that is empty initially, we select single minimum cost path $P_{\min }$ of $G_{m_{k}}^{s}$ that contains a node $\alpha$. Once $P_{\min }$ via $\alpha$ is selected, nodes of $P_{\min }$ are added to $T$, and all other redundant nodes and links are pruned from $G_{m_{k}}^{s}$. When $\alpha$ is in $T$, then we just add $G_{m_{j}}^{\alpha}$ to the set $G^{\prime}$. We repeat this process until all $G_{m_{j}}^{\alpha}$ with $\alpha \in V^{\prime}$ and $m_{j} \in D$ are considered. At the end of process, if there exist $G_{m_{j}}^{\alpha}$ of which $P_{\min }$ is not selected, single path from such $G_{m_{j}}^{\alpha}$ selected and all redundant nodes and links 


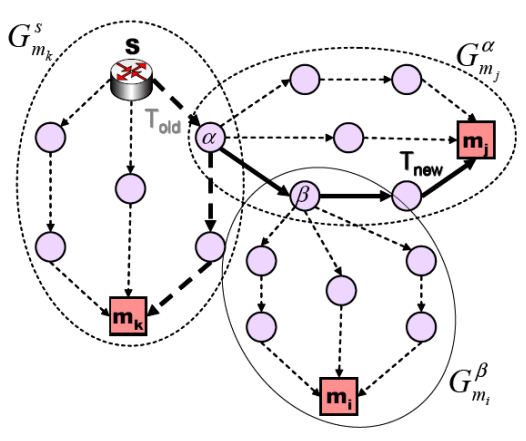

(a) MCMT basic concept

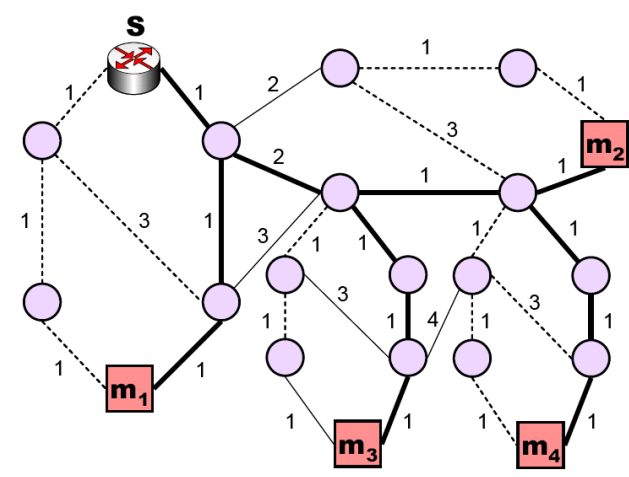

(b) A multicast tree based on MCMT

Fig. 2. e.g., MCMT algorithm

are removed. Then, the final sub-graph, $G^{\prime}$, is a tree, and spans all destinations. We strongly believe MCMT outperforms KMB in terms of tree cost. Therefore, in this paper, we propose an algorithm, Group MCMT (GMCMT), that is based on the adaptation of MCMT algorithm.

\subsection{Group MCMT Algorithm}

According to GKMB algorithm, which is based on KMB algorithm, it only considers the shortest paths between the destination nodes in multicast members set $D$ in the construction of each multicast tree. In particular, the selection of the least cost link in each iteration of the greedy strategy is made from the set of shortest paths between the multicasting members. However, if we increase the set for the greedy strategy, the final multicast trees may lead to lower cost solution for GMRP. The proposed algorithm is based on MCMT algorithm [3] and we call this algorithm Group MCMT (GMCMT). Since the MCMT algorithm finds multiple shortest paths and also considers the nodes between the group members and the relay nodes, we strongly believe that the proposed algorithm GMCMT is better than the performance of GKMB.

The GMCMT algorithm constructs a multicast tree $T_{d}$, rooted at $d \in D$, using the MCMT algorithm. If saturated edges occurs in a tree $T_{d}$, then it may imply that some trees which uses these edges will have to release them and use other alternative edges. In this case, the goal is to obtain a feasible solution with least cost possible. Since the problem of finding feasible solution for the GMRP is NP-complete, however, the determination of which set of trees to throw up the saturated edges will be a non-trivial task. In GMCMT algorithm, the alternative overhead of the current tree $T_{d}$ is compared with the alternative overhead of the most recently built tree that uses the saturated edges. The party that has the smaller alternative overhead will have to give up the saturated edges and use alternative links to get to other member nodes of $D$. The GMCMT algorithm runs in time $O\left(k^{3} n^{2}\right)$ with $|D|=k$ and $|V|=n$. 


\section{Performance Evaluations}

\subsection{Random Real Network Topology}

Random graphs of the acknowledged model represent different kinds of networks, communication networks in particular. There are many algorithms and programs, but the speed is usually the main goal, not the statistical properties. In the last decade the problem was discussed, for examples, by B. M. Waxman (1993) [7, M. Doar (1993, 1996) 89, C.-K. Toh (1993) 10, E. W. Zegura, K. L. Calvert, and S. Bhattacharjee (1996) [11], K. L. Calvert, M. Doar, and M. Doar (1997) 12, R. Kumar, P. Raghavan, S. Rajagopalan, D. Sivakumar, A. Tomkins, and E. Upfal (2000) [13]. They have presented fast algorithms that allow the generation of random graphs with different properties, in particular, these are similar to real communication networks. However, none of them have discussed the stochastic properties of generated random graphs. A. S. Rodionov and H. Choo [14] have formulated two major demands for the generators of random graph: attainability of all graphs with required properties and uniformity of distribution. If the second demand is sometimes difficult to prove theoretically, it is possible to check the distribution statistically. The method uses parameter Pe, the probability of link existence between any node pair. We use the method by Rodionov and Choo.

\subsection{Simulation Results}

We now describe some numerical results with which we compare the performance of the proposed schemes. We generate 100 different random networks for each size of 25, 100, 150, and 200. Each node in network has the probability of link existence $P_{e}=0.3$. The algorithms, GKMB and GMCMT, are implemented in C. The destination nodes are picked uniformly from the set of nodes in the network topology (excluding the nodes already selected for the destination). Moreover, the destination nodes in the multicast group, $D$, are occupied $5 \% \sim 50 \%$ of the overall nodes on the network. The link cost values in our computer experiment are selected uniformly between 1 and 10 . We simulate 1000 times $(10 \times 100=$ 1000) for each $|V|$. For the performance comparison, we implement GKMB and GMCMT in the same simulation environments.

Fig. 3 are described that GMCMT is always better than GKMB. We use the normalized surcharge, introduced in [15], of the algorithm with respect to our method defined as follows:

$$
\delta_{C}=\frac{\phi_{C}\left(T_{G K M B}\right)-\phi_{C}\left(T_{G M C M T}\right)}{\phi_{C}\left(T_{G M C M T}\right)}
$$

The enhancement is up to about $10 \% \sim 25 \%(|V|: 200)$ in terms of normalized surcharge for the GKMB tree cost. 


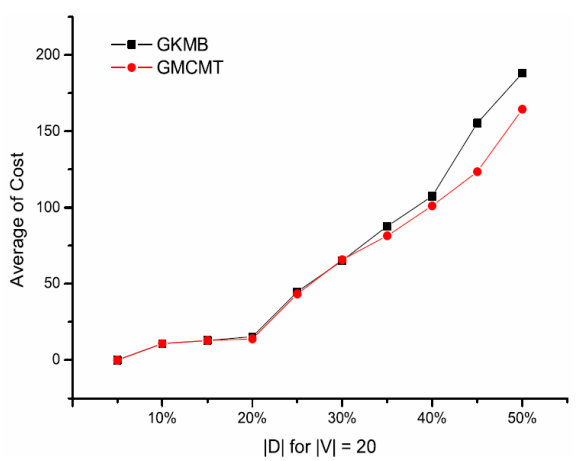

(a) $|V|: 20$ with $P_{e}: 0.3$

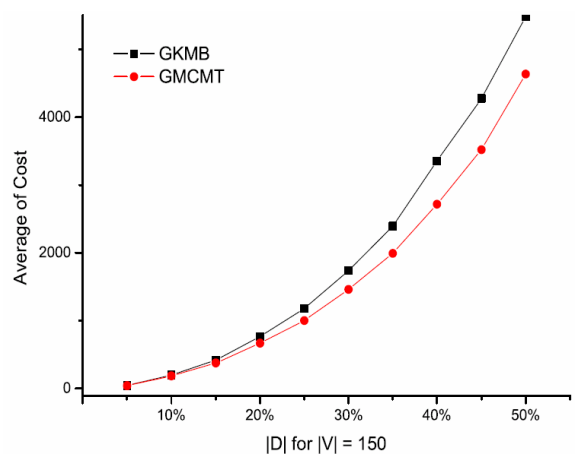

(c) $|V|: 150$ with $P_{e}: 0.3$

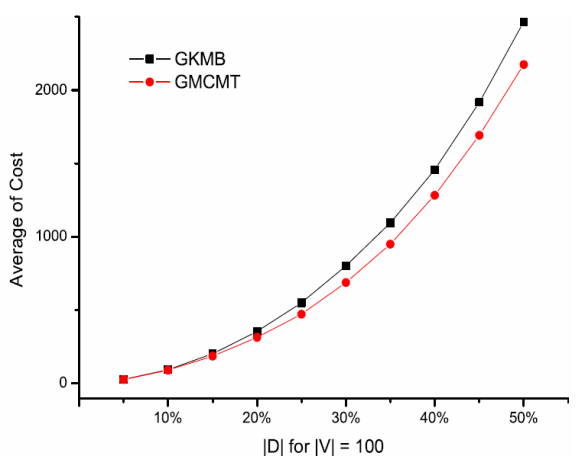

(b) $|V|: 100$ with $P_{e}: 0.3$

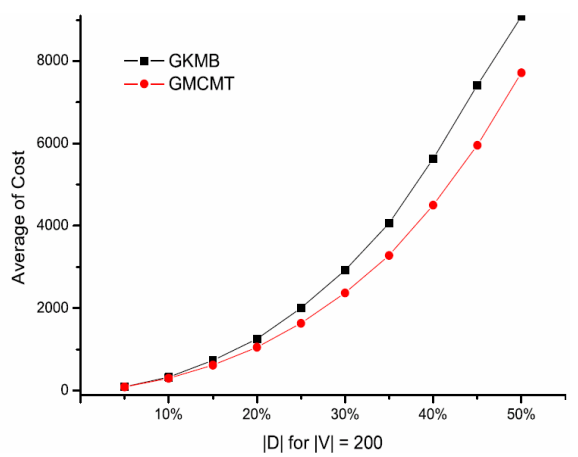

(d) $|V|: 200$ with $P_{e}: 0.3$

Fig. 3. Total tree costs

\section{Conclusion}

In this paper, we study the problem of constructing minimum cost group multicast trees with bandwidth reservations. Following that we propose a new efficient heuristic algorithm, called GMCMT, for finding low cost solutions for GMRP. Results from our computer simulation show that GMCMT algorithm performs better than GKMB algorithm in terms of total tree cost. For future work, we intend to investigate GMRP under dynamic group multicast routing. Any change of the group membership (i.e., a process quits or joins the group) would incur the re-computation of the routing trees of the group if keeping the network cost optimal after the group membership change is the primary goal. Furthermore, another future research direction is to extend the algorithm to GMRP under other kinds of constraints.

\section{Acknowledgment}

This research was supported by Ministry of Information and Communication, Korea under ITRC IITA-2006-(C1090-0603-0046). 


\section{References}

1. X. Jia and L. Wang, "A group multicast routing algorithm by using multiple minimum Steiner trees," Elsevier Computer Communications, vol. 20, pp. 750-758, 1997.

2. L. Kou, G. Markowsky, and L. Berman, "A fast algorithm for Steiner trees," Acta Informatica, vol. 15, pp. 141-145, 1981.

3. Y.-C. Bang, S.-T. Chung, M. Kim, and S.-S. Joo, "On Multicast Communications with Minimum Resources," Springer-Verlag Lecture Notes in Computer Science, vol. 3726, pp. 4-13, September 2005.

4. M. Doar and I. Leslie, "How Bad is Naive Multicast Routing?," In Proceeding of IEEE INFOCOM 93, pp. 82-89, 1993.

5. R. K. Ajuja, T. L. Magnanti, and J. B. Orlin, Network Flows: Theory, Algorithms, and Applications, Prentice-Hall, 1993.

6. R. C. Prim, "Shortest Connection Networks And Some Generalizations," Bell System Techn. J. 36, pp. 1389-1401, 1957.

7. B. W. Waxman, "Routing of multipoint connections," IEEE Journal on Selected Areas in Communications, vol. 6, no. 9, pp. 1617-1622, December 1988.

8. M. Doar, "Multicast in the ATM environment," Ph.D dissertation, Cambridge University, Computer Lab., September 1993.

9. M. Doar, "A Better Mode for Generating Test Networks," In Proceeding of IEEE GLOBECOM 96, pp. 86-93, 1996.

10. C.-K. Toh, "Performance Evaluation of Crossover Switch Discovery Algorithms for Wireless ATM LANs," In Proceeding of IEEE INFOCOM 96, pp. 1380-1387, 1996.

11. E. W. Zegura, K. L. Calvert, and S. Bhattacharjee, "How to model an Internetwork," In Proceeding of IEEE INFOCOM 96, pp. 594-602, 1996.

12. K. L. Calvert, M. Doar, and M. Doar, "Modelling Internet Topology," IEEE Communications Magazine, pp. 160-163, June 1997.

13. R. Kumar, P. Raghavan, S. Rajagopalan, D. Sivakumar, A. Tomkins, and E. Upfal, "Stochastic Models for the Web Graph," In Proceeding of the 41th IEEE Symposium on Foundations of Computer Science 2000.

14. A. S. Rodionov and H. Choo, "On Generating Random Network Structures: Connected Graphs," Springer-Verlag Lecture Notes in Computer Science, vol. 3090, pp. 483-491, September 2004.

15. V. P. Kompella, J. C. Pasquale, and G. C. Polyzoa, "Multicast routing for multimedia communications," IEEE/ACM Transactions on Networking, vol. 1, no. 3, pp. 286-292, 1993. 